Research Article

\title{
Investigation of Seismic Behavior of Clay-Pile Using Nonlinear Kinematic Hardening Model
}

\author{
Mohsen Saleh Asheghabadi (iD) and Xiaohui Cheng (iD \\ Department of Civil Engineering, Tsinghua University, Beijing 100084, China \\ Correspondence should be addressed to Xiaohui Cheng; chengxh@tsinghua.edu.cn
}

Received 16 December 2019; Revised 28 May 2020; Accepted 7 July 2020; Published 25 July 2020

Academic Editor: Wayne Yu Wang

Copyright (c) 2020 Mohsen Saleh Asheghabadi and Xiaohui Cheng. This is an open access article distributed under the Creative Commons Attribution License, which permits unrestricted use, distribution, and reproduction in any medium, provided the original work is properly cited.

\begin{abstract}
In geotechnical mediums where the bearing capacity of upper layers of soil is not suitable for use of the shallow foundations, piles are usually used as deep foundations to transfer loads to the stronger lower layers. Here, the seismic behavior of single pile and pile group constructed in saturated soft kaolin clay under three different earthquakes using Abacus 3D software is investigated. The aluminum material considering the linear elastic model has been used for the piles, and the nonlinear kinematic hardening model with Von Mises failure criterion has been considered for clay. This model can consider the soil stiffness degradation by increasing the number of cyclic loading. Three different methods have been used to calibrate the model parameters, two of them are new methods. In all calibration methods, the cyclic shear and undrained cyclic triaxial tests are used. The results obtained from the numerical analysis of the soil-pile model are in relatively good agreement with the centrifuge model results. According to the results, the variation of earthquake frequency and intensity affects the bending moment created along the pile and also the distance between piles in a pile group affects the amount of the interaction between them.
\end{abstract}

\section{Introduction}

In case of low bearing capacity of soil for use of shallow foundations, deep foundations (pile foundations) are used. Also, when the top layer or layers of soil are highly compressible or very weak so that the shallow foundation cannot be used to distribute the structural load, pile foundations are used to transfer the load to the stiffer bottom layers or bedrock. When the bedrock or stiffer bottom layers are at the deeper depth below the soil surface, the pile is used for the gradual transfer of the loads $[1,2]$. Depending on the load type, soil, and structural conditions (such as dimensions of structure, distance between piles in a pile group, distance of the structure from bedrock and soil surface, definition of interaction type between structure and surrounding soil, etc.), the piles can be used in a group or single. Due to the construction of some structures in earthquake areas, investigating the seismic behavior of piles, especially in soft soils, has become a challenge for geotechnical engineers. For instance, the performance of piles in the loose saturated soils under seismic loads is an important issue due to the effects of increasing pore water pressure in them. Decreased soil strength and stiffness due to the weak soil or liquefaction may lead to the development of large bending moment and shear forces in the pile, which may lead to pile damage.

In recent years, researchers have used various constitutive models using different software to investigate the seismic behavior of soils. Zhang et al.'s [3] study on the dynamic behavior of the pile-raft in soft clay showed that some critical factors such as peak acceleration, type of soil, and configuration of structure affect the pile behavior under the earthquake loads. In all analyses, the hyperbolic-hysteretic model was considered for soft clay. This model cannot give accurate results for the sophisticated loading conditions. Anoyatis et al. [4] have studied the kinematic response of single piles using the Winkler model which considers the pile as a flexural beam. The result of this study was that Piles kinematic response depends on a single dimensionless frequency parameter. The fundamental problem with the use of this model is to determine the stiffness of elastic springs 
used to replace the soil below the foundation. Maiorano et al. [5] and Saleh et al. [6, 7] have investigated the time-history bending moments developing during earthquakes using a linear elastic constitutive model for soil. This constitutive model cannot predict soil behavior in the plastic phase as well as in large strains. The linear Winkler and linear elastic constitutive models cannot consider the nonlinear behavior of materials and soil stiffness degradation. Patil et al. [8] conducted a numerical study on the soil behavior under seismic loads using the modified Cam-Clay model for the soil medium. This constitutive model is able to consider the soil response under the isotropic hardening loads but cannot predict the soil behavior under the cyclic kinematic hardening loading. Rayhani et al. [9] studied the seismic soilstructure interaction in clay. The linear elastic-perfectly plastic Mohr-Coulomb model was used to simulate the nonlinear soil behavior using FLAC3D. The results showed that the earthquake waves imposed to the bedrock were amplified after passing through the soil layer, especially for weak earthquake motions. This model is not a suitable model for seismic investigation of soil due to the inability to create hysteresis loops and considering the soil stiffness degradation. Moreover, although there are a number of sophisticated constitutive models such as Prevost's multi-yield model [10] and the intergranular strain anisotropy model (ISA) [11] to investigate the soil seismic behavior in the literature, due to the required extensive soil undrained triaxial test (UTC), oedometric compression test (OC), drained triaxial test (DTC), and cyclic undrained triaxial test (CUTC) for calibration of several constitutive parameters, their use is limited in practice. In this paper, a simplified but fairly comprehensive constitutive model is used to overcome the difficulties above and to better understand the dynamic response of piles under seismic loads (especially in 3D analysis). The constitutive model studied in this research for soft kaolin clay is the nonlinear kinematic hardening model [12] which is based on the Von Mises failure criterion. In the kinematic hardening, the size of the yield surface does not change but only moves in the stress space which takes the Bauschinger effect into account [13].

Calibration of constitutive model parameters is one of the most fundamental issues in using them in engineering analysis. If the appropriate parameters are not used in the model, the model prediction is accompanied by error, in such a way that a very strong and complex constitutive model may lose its efficiency due to inappropriate parameters. With the development of constitutive models, the presented constitutive models which include more parameters than traditional constitutive models are very sensitive and have a great effect on the final results. For each constitutive model, there may be several ways to calibrate its parameters. In this paper, the nonlinear kinematic hardening constitutive model and the procedure of calibrating its parameters in three different methods where second and third methods are new have been investigated. The first calibration method of parameters is based on the simulation results of the cycle shear tests and the experimental curve of Ishibashi and Zhang (cyclic shear tests curve) [14]. Second, simulation and experimental data from undrained cyclic triaxial tests are used. Third, simulation results of the cyclic shear tests and experimental data of the undrained cyclic triaxial tests are used to calibrate the parameters. The results obtained from numerical analysis using three different methods of all parameters calibration were compared with the centrifuge results [15] to investigate the seismic behavior of the pile inside the soil. It is worth noting that for the first time the seismic behavior of the pile group in the soft soil has been investigated using the nonlinear kinematic hardening model. The soil studied in this research is saturated soft kaolin clay and all analyses are performed using the finite element method in 3D Abaqus. Three types of earthquakes with different peak ground acceleration of $0.07 \mathrm{~g}, 0.1 \mathrm{~g}$, and $0.22 \mathrm{~g}$ considering tied degrees of freedom (TDOF) boundary were used and the linear elastic model is considered for aluminum piles and caps.

\section{Constitutive Model and Calibration}

2.1. Constitutive Model. Soils are among the engineering materials that exhibit complex behavior due to different loading conditions. One of the main factors of this complexity is the multiphase of soils (soil particle, air, and water) compared with other engineering materials as well as their high compressibility. Some of the features that a constitutive model has to offer for soils include nonlinearity of stressstrain relation and irreversibility of part of deformation (plastic strain), considering the phenomena of dilation and contraction, nonisotropic, and the creation of hysteresis loops in loading and unloading cycles. One of the constitutive models for studying the behavior of soils under cyclic loading is the isotropic/kinematic hardening model $[15,16]$. Isotropic hardening is the simplest hardening model, and this model cannot take Bauschinger's effect observed in experimental tests, while kinematic hardening takes Bauschinger's effect [17].

In geotechnical analyses, the use of the nonlinear kinematic hardening model with Von Mises failure criterion for clay soil in undrained conditions (because of the independence of pressure) is appropriate, but it should be modified for sand soil $[18,19]$.

The general relation for the evolution of stress is expressed as follows:

$$
\sigma=\sigma_{\mathrm{s}}+\alpha
$$

where $\alpha$ and $\sigma_{\text {s }}$ are back-stress and instantaneous yield surface size. Back-stress refers to the kinematic evolution of the yield surface.

The isotropic part of this model relates to the changing of the yield surface size expressed below (this is a function of the equivalent plastic strain):

$$
\sigma_{\mathrm{s}}=\sigma_{0}+Q_{\infty}\left(1-e^{-b \varepsilon_{\mathrm{pl}}}\right)
$$

where $\sigma_{0}$ is the initial size of the yield surface, $\varepsilon_{\mathrm{pl}}$ is the equivalent plastic strain, and $Q_{\infty}$ and $b$ are the model parameters where $Q_{\infty}$ refers to the evolution of the yield surface and $b$ is the rate of this evolution. If the size of the yield surface remains constant $\left(Q_{\infty}=0\right.$ and $\left.\sigma_{\mathrm{s}}=\sigma_{0}\right)$, then 
only the yield surface displacement will occur. In this case, the combined hardening model reduces to a nonlinear kinematic hardening model (as assumed in the paper).

As described above, the parameter $\sigma_{\mathrm{s}}=\sigma_{0}$ is the initial yield stress of the model considering the nonlinear kinematic hardening model and the plastic behavior of soil is controlled by this parameter which is the fraction of the effective maximum yield stress and is expressed as follows:

$$
\sigma_{0}=\lambda \sigma_{\mathrm{y}} .
$$

In equation (3), $\lambda$ is a scaler and $\sigma_{\mathrm{y}}$ effective maximum yield stress.

The evolution equation of kinematic hardening can be integrated analytically to give

$$
\alpha=\frac{C}{\gamma_{\mathrm{o}}}\left[1-\exp \left(-\gamma_{\mathrm{o}} \varepsilon_{\mathrm{pl}}\right)\right],
$$

here, $C$ and $\gamma_{\mathrm{o}}$ are kinematic hardening parameters.

$C$ is the initial kinematic hardening modulus and $\gamma_{\mathrm{o}}$ refers to the $C$ reduction. The amount of translation of the yield surface is determined by the parameter $C$. The parameter $C$ is obtained as follows [20-22]:

$$
\left.\frac{\partial \alpha}{\partial \varepsilon^{\mathrm{pl}}}\right|_{\mathcal{E}^{\mathrm{pl}}} \longrightarrow 0=E .
$$

Figure 1 shows the one-dimensional representation of the kinematic hardening model. $\alpha_{\mathrm{s}}$ is the magnitude of $\alpha=$ $C / \gamma_{\mathrm{o}}$ at large plastic strains.

2.2. Calibration of Parameters. Using soil constitutive models is a method for describing soil behavior and strains caused by stresses. These models are expressed according to the physical nature and available experiences from the environment in terms of mathematical-based equations. Regardless of the type of calibration model, its parameters are considered as one of the most important steps in the use of these models, because if the parameters are not realistic, the results of predicting soil behavior will not be appropriate with the model. Therefore, any constitutive model before being used must be calibrated based on the results of the experimental tests. So far, various methods have been proposed to calibrate the parameters of constitutive models. In traditional calibration procedures, levels of stress and strain in certain modes are used by performing specific experimental tests on soil. Sometimes traditional methods of calibration of constitutive model parameters cannot predict the soil behavior completely. In this research, the results of undrained cyclic triaxial tests [23] were used to obtain the parameter $C$ and three different methods have been used to calibrate the kinematic hardening parameters including $\lambda$, $\gamma_{\mathrm{o}}$, and $\sigma_{0}$ for soft kaolin clay and then the results of them are compared with each other.

The first method is to plot the shear modulus $(G)$ in terms of shear strain $(\gamma)$ using a cyclic shear test simulation and compare it with published curves of Ishibashi and Zhang [14]. The researchers used this method in previous studies to calibrate the parameters of the kinematic hardening model $[18,24,25]$. The second method is to plot Young's modulus

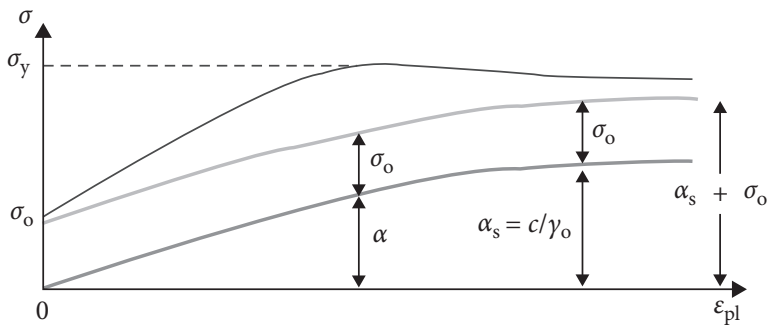

Figure 1: Simplified 1D representation of the hardening.

$(E)$ versus the axial strain $\left(\varepsilon_{1}\right)$ using the experimental results of undrained cyclic triaxial tests and compare them with the results of the simulation of these tests. In the third method, the calibration of the parameters is presented using the experimental data of undrained cyclic triaxial tests and the simulation results of the triaxial cyclic shear tests. The second and third methods are the new methods presented in this study. In the following, the procedure to obtain the parameters required in this constitutive model is fully described.

2.2.1. Determination of $C$. Undrained cyclic triaxial tests data can be used to obtain the undrained Young's modulus $\left(E_{u}\right)$. A number of these experimental tests $(\mathrm{C} 1-\mathrm{C} 8$ and C32-C36) were performed by Wichtmann [23] under the stress control conditions. In all experimental tests, the reduction of soil stiffness and increase of damping caused by the soil plasticity due to increasing the number of cyclic loading and unloading are shown.

First, Young's modulus corresponding to the second and fifth cycles of each undrained cyclic triaxial test $\left(q-\varepsilon_{1}\right)$ is calculated. Then, using this data, the curve of undrained Young's modulus can be plotted versus the axial strain amplitude $\left(E_{u}-\varepsilon_{1}\right)$ to derive the undrained Young's modulus. Figure 2 shows the values of normalized undrained Young's modulus vs. strain amplitude based on the experimental data of undrained cyclic triaxial tests. In this figure, undrained Young's modulus $\left(E_{u}\right)$ was normalized by the equation of void ratio $\left(f_{E_{u}, e}\right)$ that is defined as follows:

$$
f_{E_{u}, e}=\frac{(4.4-e)^{2}}{(1+e)},
$$

where $e$ is the void ratio of soil. Also, in Figure 2, simulation results of the undrained cyclic triaxial tests are presented in order to calibrate the parameter $\lambda$. The procedure to obtain the parameter $\lambda$ using these data is described in Section 2.2.2.

According to the experimental data of Figure 2, the decrease in $E_{u}$ with increasing strain amplitude $\left(\varepsilon_{1}^{\text {ampl }}\right)$ can be described by [26]

$$
\frac{E_{u}}{E_{u_{\max }}}=f_{E_{u} \text { ampl }}=\frac{1}{1+\left(\varepsilon_{1}^{\text {ampl }} / 3 * 10^{-4}\right)^{0.9} .}
$$

With an extrapolation on the data of Figure 2, equation $E_{u_{\max }} \approx 30^{*} f_{E_{u}, e}$ is obtained. So, using equations (6) and (7), the values of Young's modulus in different strain points are obtained from the following equation: 


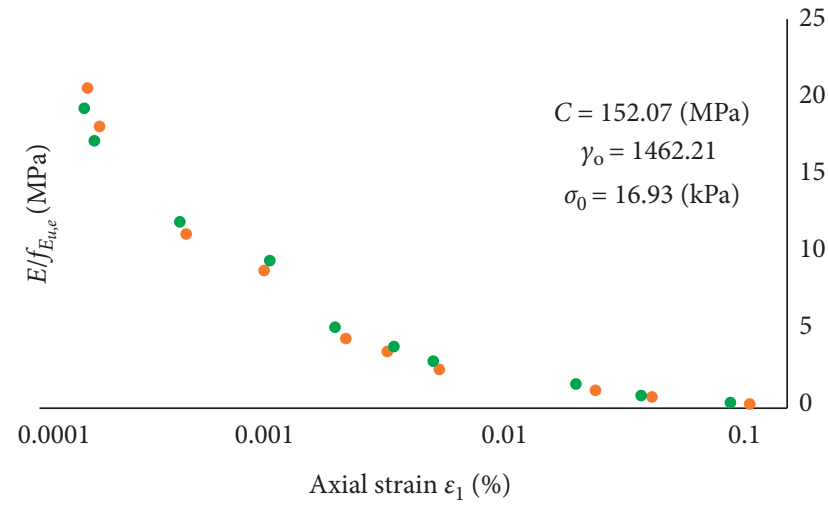

- Experimental data (undrained cyclic triaxial test) [23]

- Simulation data (undrained cyclic triaxial test)

Figure 2: Calibration of parameters $C$ and $\lambda$.

$$
C=E_{u_{\max }}=30 * f_{E_{u}, \mathrm{ampl}} * f_{E_{u}, e}
$$

Using the results obtained from the undrained cyclic triaxial tests at very small strains $\left(\varepsilon_{1}^{\text {ampl }}<10^{-6}\right)$, the value of $C$ can be calculated.

$G_{\max }$ is obtained using the maximum undrained Young's modulus by $G_{\max }=E_{u_{\max }} / 2\left(1+v_{\max }\right)$. In this equation, $v_{\max }$ is the Poisson ratio.

2.2.2. The First Calibration Method of Parameters $\lambda, \sigma_{0}$, and $\gamma_{o}$. It may be necessary to perform several different tests to calibrate all parameters of a constitutive model. For example, monotonic triaxial tests are required in order to obtain other clay parameters such as undrained shear strength $\left(S_{\mathrm{u}}\right)$. A number of undrained monotonic triaxial tests on kaolin clay by Wichtmann were performed [23]. In this paper, for instance, the results of the cyclic and monotonic experimental tests with an effective mean pressure of $200 \mathrm{kPa}$ were used to calculate and explain the method of calibrating soil parameters. The curve of deviatoric stress vs. axial strain of the monotonic test is used to derive the undrained shear strength $\left(S_{\mathrm{u}}\right)$ using

$$
S_{\mathrm{u}}=\frac{\sigma_{1}-\sigma_{3}}{2}=\frac{q}{2}
$$

where $\sigma_{1}$ is maximum mean stress and $\sigma_{3}$ is minimum mean stress.

The effective maximum yield stress considering pure shear test is calculated as follows (Appendix):

$$
\sigma_{y}=\sqrt{3} S_{\mathrm{u}} \text {. }
$$

The first calibration method of the parameter $\lambda$ is based on the plasticity index (PI) of clay. If the cyclic shear or cyclic triaxial test data is not available, this method can be used to calibrate the parameter $\lambda$. First, simulation of the cyclic shear test is performed under the strain control conditions for different strain amplitudes; then, for each test the value of the shear modulus corresponding to the shear strain is obtained based on the stabilized cycle. Finally, according to the data obtained from the simulation of the cyclic shear tests and the experimental curve of Ishibashi and Zhang using equations (11)-(14) [14], the curve of normalized shear modulus $\left(G / G_{\max }\right)$ versus shear strain $(\gamma)$ diagram is plotted in order to calibrate the parameter $\lambda$ (Figure 3 ). In clay soils, the maximum shear modulus $\left(G_{\max }\right)$ depends on the soil plasticity index [27]. If the PI increases, $G / G_{\max }$ vs. $\gamma$ tends to locate higher. Therefore, the shear modulus and damping ratio are strongly influenced by the plastic index in clay soils, so that the value of the damping ratio decreases with increasing the plasticity index while the shear modulus increases:

$$
\begin{aligned}
& \frac{G}{G_{\max }}=K\left(\gamma, I_{p}\right) \bar{\sigma}_{0}^{m\left(y, I_{p}\right)-m_{0}}, \\
& m\left(\gamma, I_{p}\right)-m_{0}=0.272\left[1-\tanh \left\{\ln \left(\frac{0.000556}{\gamma}\right)^{0.4}\right\}\right] e^{-0.0145 I_{p}^{1.3}} \\
& K\left(\gamma, I_{p}\right)=0.5\left[1+\tanh \left\{\ln \left(\frac{0.000102+n\left(I_{p}\right)}{\gamma}\right)^{0.492}\right\}\right],
\end{aligned}
$$

After calculating the above parameters, the initial size of the yield surface can be defined according to equation (3) $\left(\sigma_{0}=\lambda \sigma_{\mathrm{y}}\right)$.

The effective maximum yield stress in kinematic hardening model is obtained as follows:

$$
\sigma_{\mathrm{y}}=\frac{C}{\gamma_{\mathrm{o}}}+\sigma_{0} .
$$

From equations (10) and (15), the parameter $\gamma_{\mathrm{o}}$ which determines the rate of decrease of the kinematic hardening modulus is obtained according to the following equation:

$$
\gamma_{\mathrm{o}}=\frac{C}{\sigma_{\mathrm{y}}-\sigma_{0}}=\frac{C}{\sqrt{3} S_{u}-\sigma_{0}} .
$$

2.2.3. The Second Calibration Method of Parameters $\lambda, \sigma_{0}$, and $\gamma_{o}$. The second method of calibrating the parameters of the kinematic hardening model is to use the curve of normalized undrained Young's modulus versus axial strain $\left(\left(E_{u} / f_{E, e}\right)-\left(\varepsilon_{1}\right)\right)$ using experimental and numerical data. Parameters of constitutive models of engineering materials are often obtained by comparing experimental results and numerical simulations using the finite element [28]. Therefore, after performing the simulation of undrained cyclic triaxial tests (deviatoric stress $(q)$ vs. axial strain $\left(\varepsilon_{1}\right)$ ) under the stress control conditions, the Young's modulus 


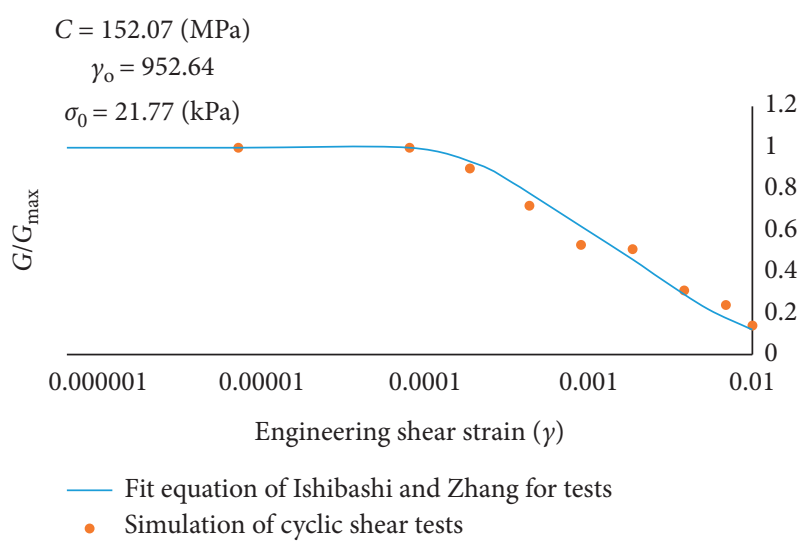

Figure 3: Comparison of FE-computed $G-\gamma$ curve with the experimental curve of Ishibashi and Zhang [14].

value corresponding to the second and fifth cycles is calculated for each test or stress amplitude. Then, the curve of normalized undrained Young's modulus versus axial strain is plotted using numerical and experimental data to calibrate the parameter $\lambda$ (see Figure 2). It is worth noting that all cycles of undrained triaxial cyclic tests can be used to calibrate the parameter $\lambda$, but the second and fifth cycles of numerical results are used in this paper because previous researchers $[23,29]$ have used the second and fifth cycles to draw Young's modulus versus axial strain (see Figure 2).

In the second method of calibrating the parameters, there is no need to perform the cyclic shear tests, and only by performing the cyclic triaxial tests can the calibration be performed. In this method, all cyclic cycles can be used and there are no restrictions in this regard, and also, it is not necessary to know the plasticity index (PI) of soil. Therefore, by having cyclic triaxial test data and drawing curve of undrained Young's modulus versus axial strain, the parameters of the constitutive model can be easily and accurately calibrated.

The effective maximum yield stress $\left(\sigma_{\mathrm{y}}\right)$ (which is related to the second invariant of the stress deviatoric tensor) for undrained clay with consideration of the triaxial test condition is obtained according to the following:

$$
J_{2}^{\prime}=\frac{1}{6}\left[\left(\sigma_{1}-\sigma_{2}\right)^{2}+\left(\sigma_{2}-\sigma_{3}\right)^{2}+\left(\sigma_{3}-\sigma_{1}\right)^{2}\right],
$$

where $J_{2}^{\prime}$ is the second invariant of the deviatoric stress tensor and $\sigma_{1}, \sigma_{2}$, and $\sigma_{3}$ are the principle stresses. In the triaxial compression test $\sigma_{2}=\sigma_{3}$, which means that equation (17) becomes

$$
J_{2}^{\prime}=J_{2 y}^{\prime}=\left.\frac{1}{3}\left(\sigma_{1}-\sigma_{3}\right)^{2}\right|_{\text {yielding condition }},
$$

where

$$
\sigma=\sigma_{\mathrm{y}}=\sqrt{J_{2 \mathrm{y}}^{\prime}} .
$$

From equations (18) and (19), we obtain

$$
\sigma_{\mathrm{y}}=\sqrt{\frac{1}{3}\left(\sigma_{1 \mathrm{y}}-\sigma_{3 \mathrm{y}}\right)^{2}} \text {. }
$$

The undrained shear strength of clay can be calculated by

$$
S_{u}=\frac{\left(\sigma_{1 \mathrm{y}}-\sigma_{3 \mathrm{y}}\right)}{2} \text {. }
$$

From equations (20) and (21), we obtain

$$
\sigma_{\mathrm{y}}=\frac{2 \sqrt{3}}{3} S_{\mathrm{u}} \text {. }
$$

After calculating the above parameters, the initial size of the yield surface can be defined according to equation (3) $\left(\sigma_{0}=\lambda \sigma_{\mathrm{y}}\right)$.

From equations (22) and (15), the parameter $\gamma_{\mathrm{o}}$ which determines the rate of decrease of the kinematic hardening modulus is obtained according to the following equation:

$$
\gamma_{\mathrm{o}}=\frac{C}{\sigma_{\mathrm{y}}-\sigma_{0}}=\frac{C}{(2 \sqrt{3} / 3) S_{\mathrm{u}}-\sigma_{0}} .
$$

2.2.4. The Third Calibration Method of Parameters $\lambda, \sigma_{0}$, and $\gamma_{o}$. In this paper, the third calibration method of the kinematic hardening model parameters is based on the experimental results of the undrained cyclic triaxial tests [23] and simulation results of the cyclic shear tests. In other words, the model parameters can be obtained by using the curve of shear modulus $\left(G / G_{\max }\right)$ vs. shear strain $(\gamma)$ based on the data obtained from both experimental tests and numerical analysis. First, the curve of shear modulus-shear strain $\left(G / G_{\max }-\gamma\right)$ (by using results of experimental and simulation of the undrained cyclic triaxial tests) is plotted based on data obtained from the undrained cyclic triaxial tests using Figure 2; then, the values of the shear modulus of stabilized cycles of the cyclic shear tests at different strain amplitudes are calculated in order to calibrate the parameter $\lambda$. Figure 4 shows the curve of shear modulus vs. shear strain based on the undrained cyclic triaxial tests data, the numerical simulation of the cyclic shear tests, and equation (7). Therefore, based on this graph and the corresponding equations, the parameter $\lambda$ of the constitutive model is obtained. In the third method of calibrating the parameters, if the cyclic shear tests data is not available, the constitutive model parameters can be obtained by simulating the cyclic shear test and using the cyclic triaxial tests data. So there is no limitation to this method. It should be noted that a soil element was used in the simulation of the undrained cyclic triaxial tests in all three calibration methods because the results presented in the experimental models are on a soil element.

After determining the parameters $S_{\mathrm{u}}, \sigma_{\mathrm{y}}$, and $\lambda$, the initial size of yield surface can be defined according to equation (3) $\left(\sigma_{0}=\lambda \sigma_{\mathrm{y}}\right)$ and the parameter $\gamma_{\mathrm{o}}$ is calculated by equation (16).

\section{Model Description}

There are several methods [30-32] to consider boundary conditions in seismic analysis. In this paper, tied degrees of freedom (TDOF) boundary [33] around all the pile-soil models were used to prevent the earthquake from returning 


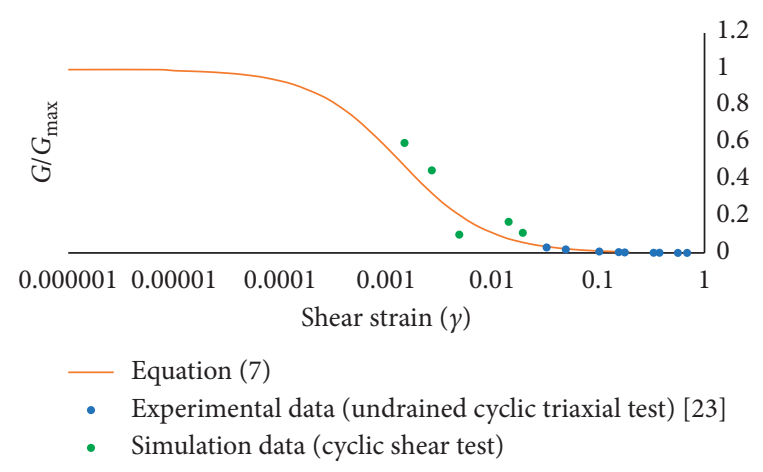

FIGURE 4: Calibration of kinematic hardening model for clay against the $G-\gamma$ curve of undrained cyclic triaxial test.

to the model for simulating the laminate box. The type of elements used for the soil is C3D8RP and the following equations (equations 24 and (25)) [34] are used to control the mesh dimensions. Therefore, the mesh dimensions are considered small enough to achieve logical answers because in dynamic analysis the size of the meshes is one of the vital factors that affects the responses. Figure 5 shows the 3 -dimensional finite element model of the soil-pile-raft system.

$$
\Delta x_{\max } \leq\left(\frac{1}{6} \sim \frac{1}{8}\right) \lambda_{\min }
$$

where $\lambda_{\min }$ is the wavelength of the minimum shear wave velocity which is obtained from the following equation:

$$
\lambda_{\text {min }}=\frac{V_{\text {min }}}{f_{\text {min }}},
$$

where $V_{\text {min }}$ is the minimum of shear wave velocity and $f_{\min }$ is the highest frequency of the input wave.

The explicit solution scheme in Abaqus is adapted in this paper for the time-step increment. In Abaqus, the Courant number must satisfy the following equation in order for the solution scheme to be stable and to converge the results [35]:

$$
C_{n}=\frac{V_{\mathrm{o}}^{*} \Delta t}{\Delta x_{\min }} \leq 1,
$$

where $V_{\mathrm{o}}$ is the wave propagation velocity and $\Delta x_{\min }$ and $\Delta t$ are the shortest element length and time increment, respectively. In this paper, considering the wave propagation velocity and shortest element length, a time-step increment of $1^{*} 10^{-5} \mathrm{~s}$ is considered.

Geostatic stresses have been considered in the models before applying the earthquake load to the bedrock. These stresses are considered in the geotechnical medium to verify that the initial geostatic stress field is in equilibrium with applied loads and boundary conditions by using the equation $\sigma^{\prime}=\gamma^{\prime} h$, where $\gamma^{\prime}$ and $h$ are the effective unit weight $\left(\mathrm{KN} / \mathrm{m}^{3}\right)$ and depth of soil $(\mathrm{m})$, respectively. Equation $K_{0}=$ $(0.95-\sin \phi)$ is used for Earth pressure or lateral coefficient at rest. The soil used in this study is soft kaolin clay. This type of soil is used in most research works (centrifuge and numerical studies). The dimensions of the model used in the numerical analysis are $11 \mathrm{~m}$ (height) ${ }^{*} 11.40 \mathrm{~m}$ (width) *

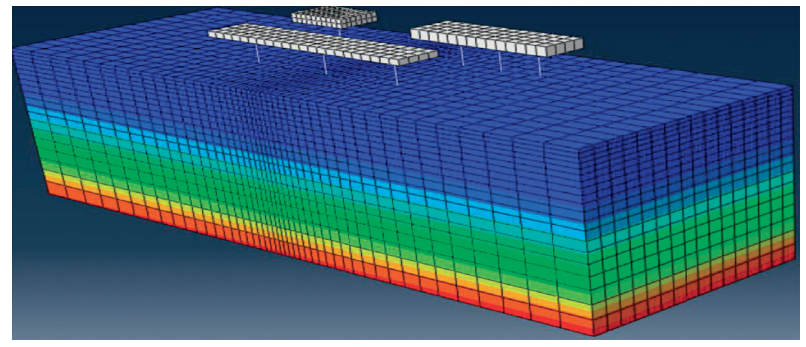

FIGURE 5: 3D finite element model of the soil-pile-raft model.

$32.25 \mathrm{~m}$ (length). The general properties of soil are presented in Table 1 . The values of the Rayleigh coefficients ( $\alpha$ and $\beta$ ) are obtained from frequency analysis for the models. In this paper, a single pile, two pile groups of $3 * 1$ (vertical circular piles), and one cap on the piles were used. The piles are made of aluminum; their characteristics are shown in Table 1. Table 2 shows the values of the kinematic hardening model parameters based on the three calibration methods described in Section 2.2.

The length of all the piles is $10.5 \mathrm{~m}$ and their inner and outer diameters are $0.75 \mathrm{~m}$ and $0.70 \mathrm{~m}$, respectively. The distance between the piles is 2 meters (pile group 1) and 4 meters (pile group 2). Cap thickness in the single pile and pile group 1 is $0.635 \mathrm{~m}$ and in pile group 2 is $0.39 \mathrm{~m}$. The dimensions and position of the piles and caps are shown in Figure 6. All dimensions are in meters except cross section $X-X$ which is in $\mathrm{mm}$ based on centrifuge model (outer diameter (cross section $X-X$ ) is $15 \mathrm{~mm}$ in centrifuge model or $0.75 \mathrm{~m}$ in prototype model). The dimensions of the soil model and the location of the piles in the clay are also shown in Figure 7. Figure 7(b) illustrates the points in the soil (P1-P5) and on the pile foundations $(\mathrm{C} 1-\mathrm{C} 2)$ where the acceleration response is measured.

Three different earthquakes with the intensity of $0.07 \mathrm{~g}$, $0.1 \mathrm{~g}$, and $0.22 \mathrm{~g}$ were used to investigate the effect of the earthquake intensity on the seismic behavior of piles. Table 3 shows the properties of input motion to the models. The acceleration-time curves of the earthquakes which applied to the bedrock are presented in Figure 8.

\section{Results and Discussion}

Due to the complexity of investigating the seismic behavior of pile in clay soils compared to sandy soils and also fewer studies on clay soils, this paper has attempted to use different methods of calibrating constitutive model parameters to achieve more realistic results. In this section, the results of the numerical method using parameters obtained from all three different calibration methods are compared with the results of the centrifuge model [15]. Although the results of all three calibration methods were close to the results of the centrifuge model, the difference between results of the first and second calibration methods with the centrifuge model was less than the differences between the results of the third method with the centrifuge model results. All analysis were 
TABLE 1: Properties of soft clay and aluminum.

\begin{tabular}{lcccccccc}
\hline Type of material & $\gamma\left(\mathrm{kg} / \mathrm{m}^{3}\right)$ & $G_{0}(\mathrm{MPa})$ & $E(\mathrm{MPa})$ & $\nu$ & $V_{\mathrm{s}}\left(\mathrm{m} / \mathrm{s}^{2}\right)$ & $S_{\mathrm{u}}(\mathrm{kPa})$ & $\alpha$ & $\beta$ \\
\hline Soft kaolin clay & 1623 & 13.146 & 39.176 & 0.49 & 90 & 8 & 1.9312 \\
Aluminum & 2700 & - & 70 & 0.3 & - & - & - & - \\
\hline
\end{tabular}

TABLE 2: Mechanical properties of soft clay based on the three different calibration methods.

\begin{tabular}{lccccc}
\hline Method no. & $\sigma_{\mathrm{y}}(\mathrm{kPa})$ & $\sigma_{0}(\mathrm{kPa})$ & $C(\mathrm{MPa})$ & $\lambda$ & $\gamma_{\mathrm{o}}$ \\
\hline First method & 13.86 & 3.74 & 39.176 & 0.27 & 3871.15 \\
Second method & 9.24 & 2.31 & 39.176 & 0.25 & 5653.1 \\
Third method & 13.86 & 4.16 & 39.176 & 0.3 & 4038.76 \\
\hline
\end{tabular}

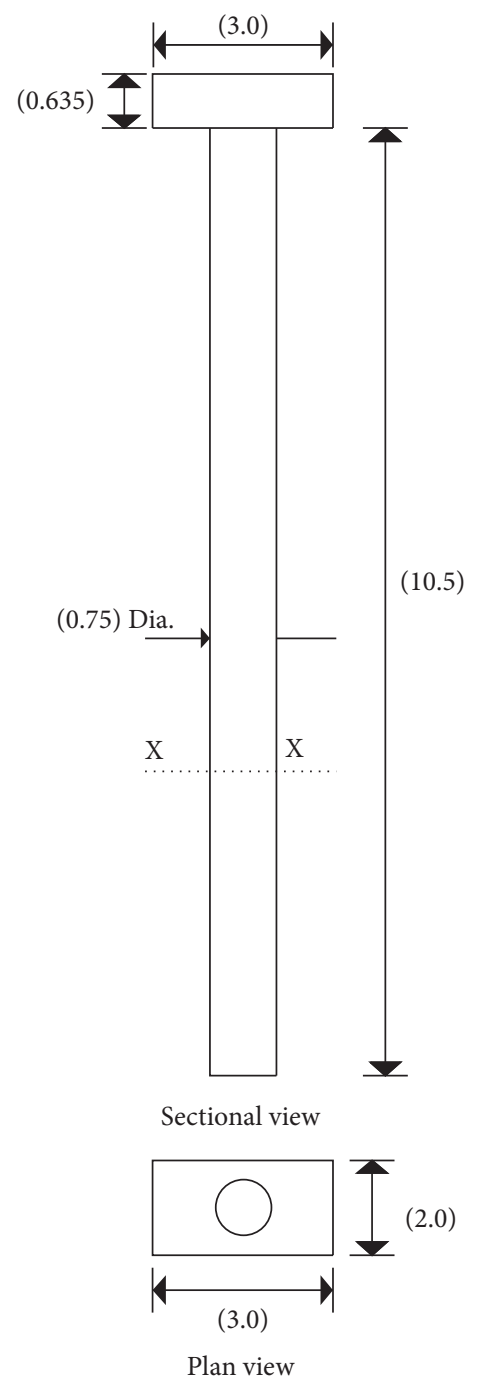

(a)

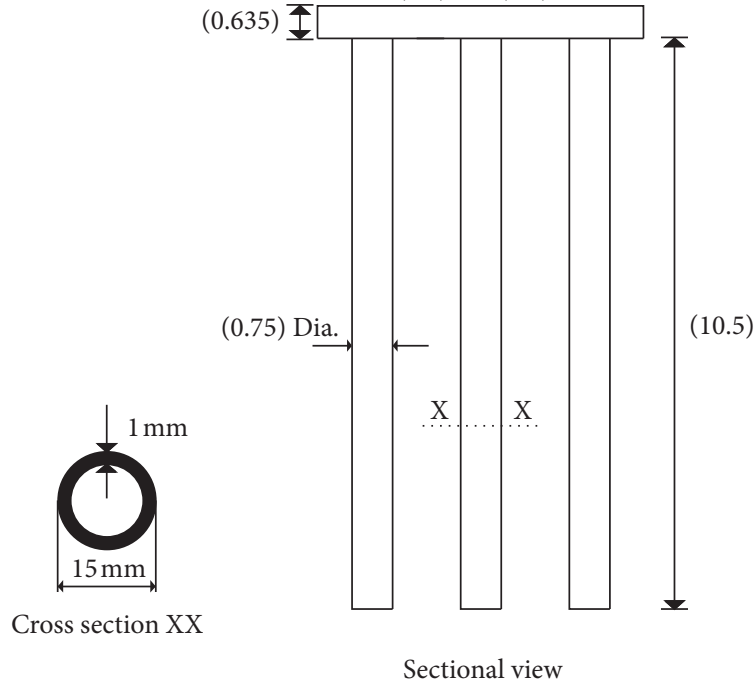

Cross section XX
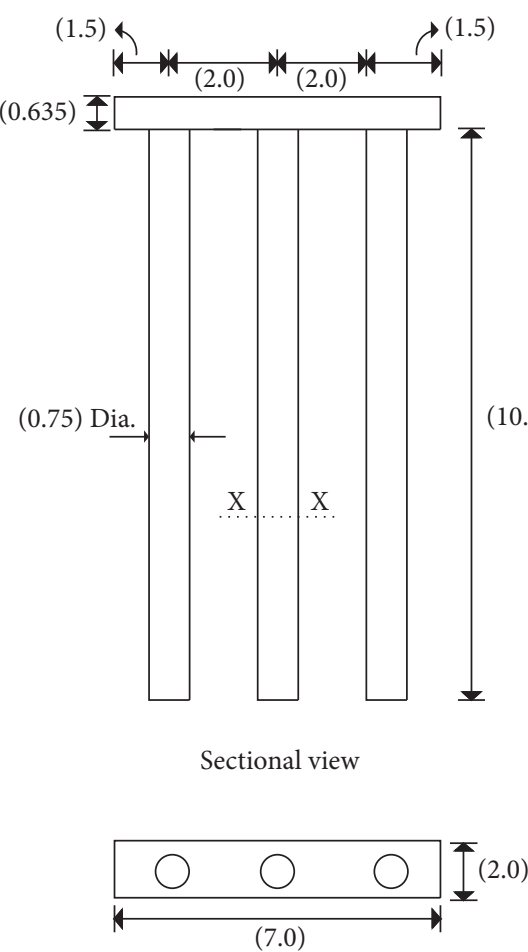

Plan view
10.5)

4038.76 


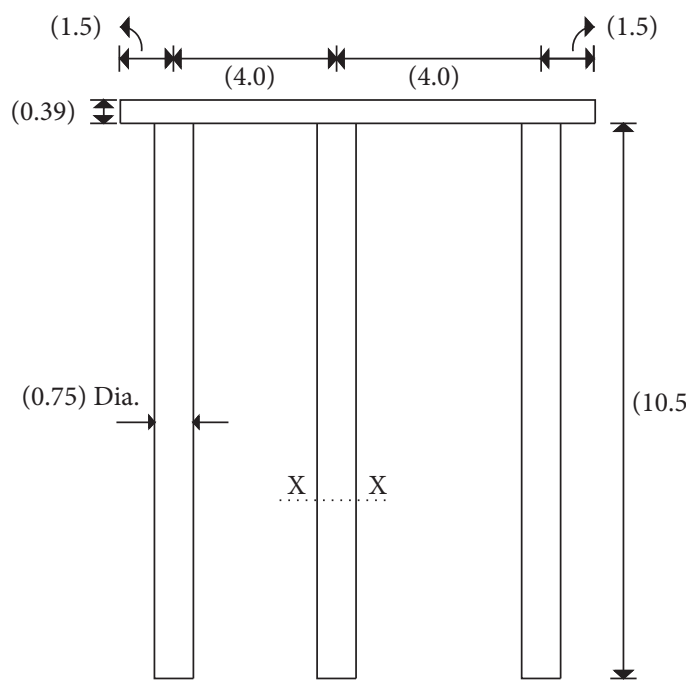

Sectional view

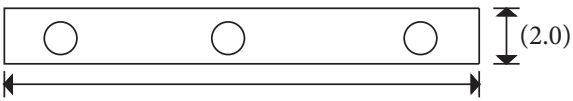

(11.0)

Plan view

(c)

Figure 6: Pile and raft: (a) single pile, (b) pile group-1, and (c) pile group-2.

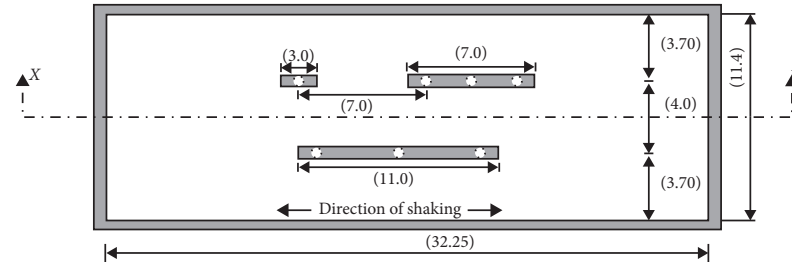

(a)

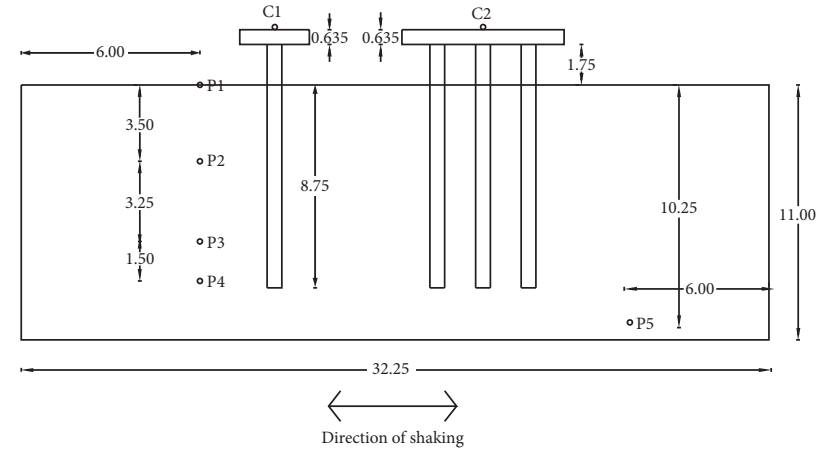

(b)

Figure 7: Soil-pile-raft system: (a) plan view of the model, (b) cross section along the plane (all dimensions are in meters).

TABle 3: Properties of earthquakes.

Earthquake no. Frequency $(\mathrm{Hz}) \quad$ Peak ground acceleration $(\mathrm{g})$

\begin{tabular}{lll}
\hline Input motion-1 & 0.6 & 0.07 \\
Input motion-2 & 0.8 & 0.10 \\
Input motion-3 & 1.2 & 0.22 \\
\hline
\end{tabular}

performed using 3D Abaqus software by considering the nonlinear kinematic hardening model for soft kaolin clay.

Different frequencies cause different responses in the soil; especially at high frequencies, the amplitude of the soil response is suddenly increased relative to the input wave amplitude. Note that the effect of plasticity at low vibration frequencies is quite tangible and decreases with increasing vibration frequency. Here, three earthquakes with three different frequencies $(0.6 \mathrm{~Hz}, 0.8 \mathrm{~Hz}$, and $1.2 \mathrm{~Hz})$ were used to investigate the effect of earthquake frequency on soil response. Figure 9 shows the comparison of the numerical analysis results with the results of the centrifuge model. In this figure, the acceleration response of clay at different depths under the low-frequency earthquake $(0.6 \mathrm{~Hz})$ is investigated. As the results show, the acceleration response decreases with increasing soil depth, so that the maximum acceleration response is on the soil surface $(0.084 \mathrm{~g})$. That is, the clay amplifies the seismic waves at low frequencies. So, 


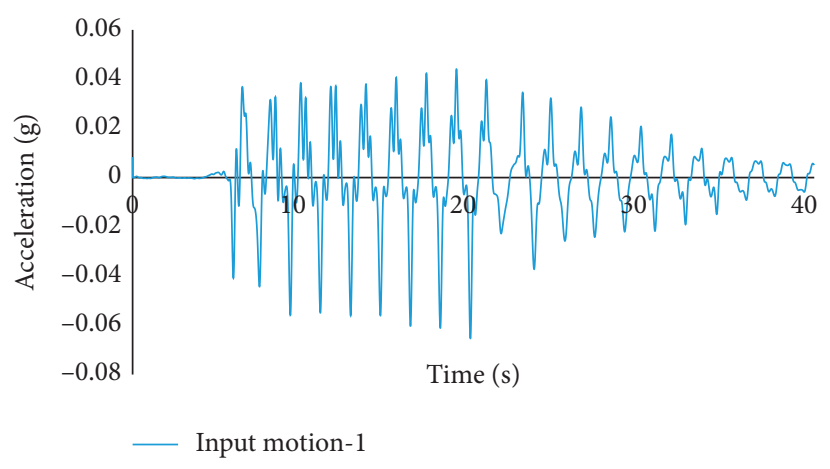

(a)

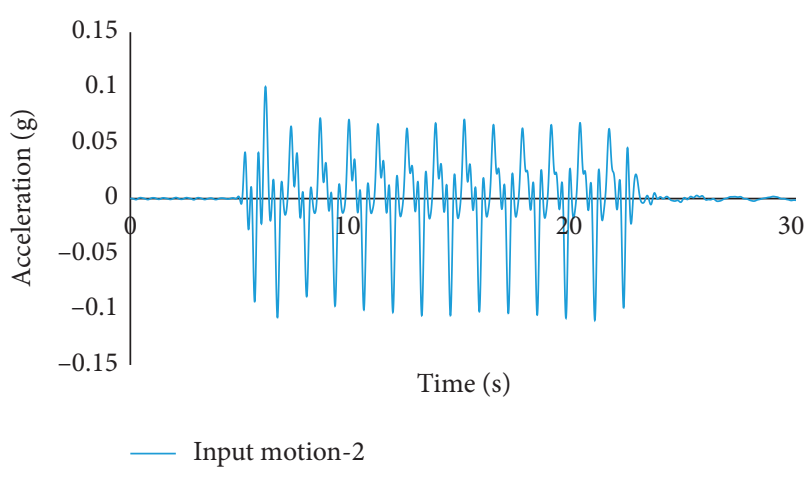

(b)

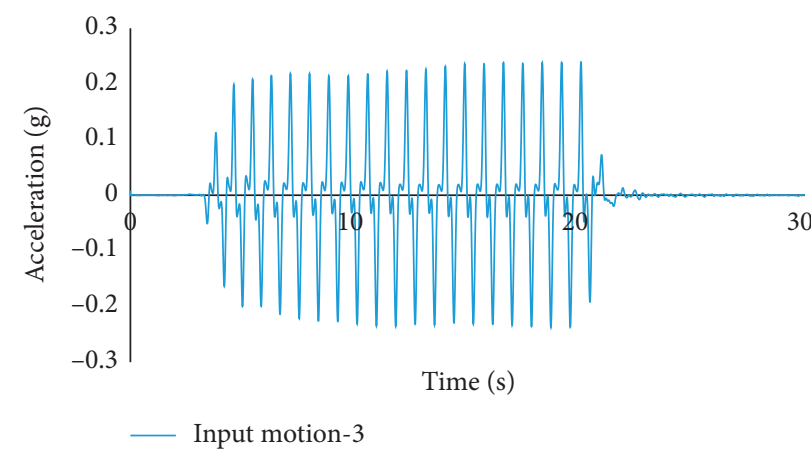

(c)

FIGURE 8: Input base accelerations: (a) input motion-1, (b) input motion-2, and (c) input motion-3.

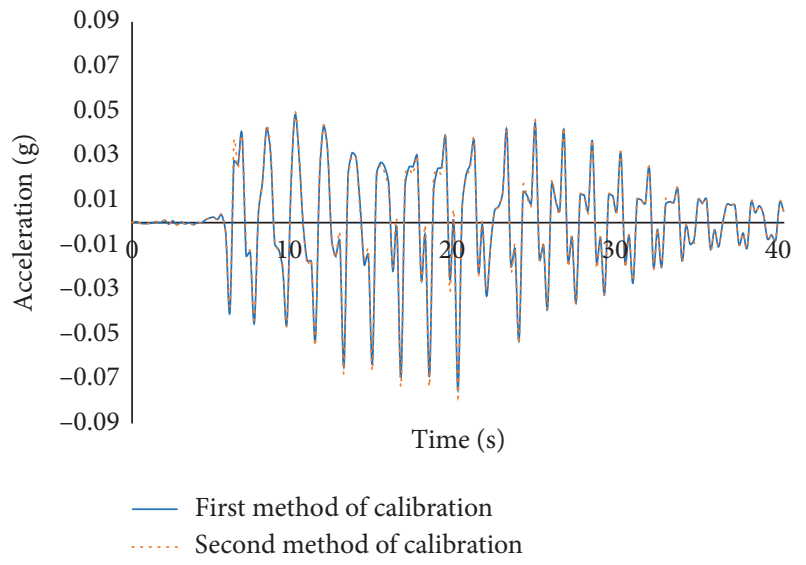

(a)

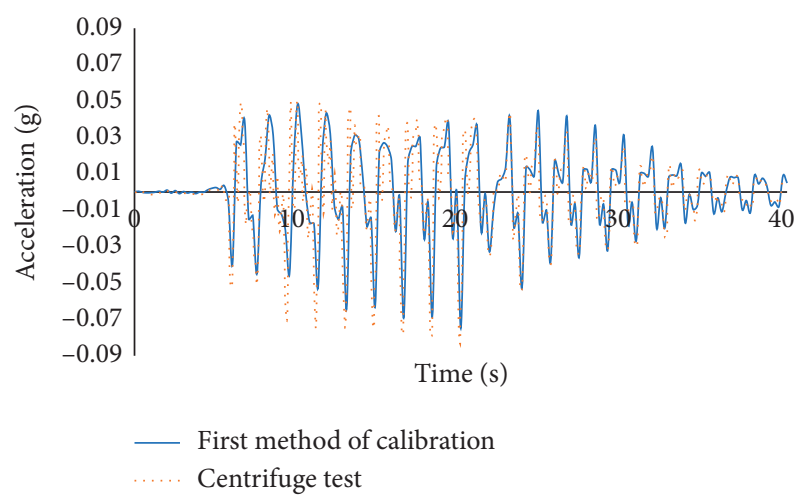

(c)

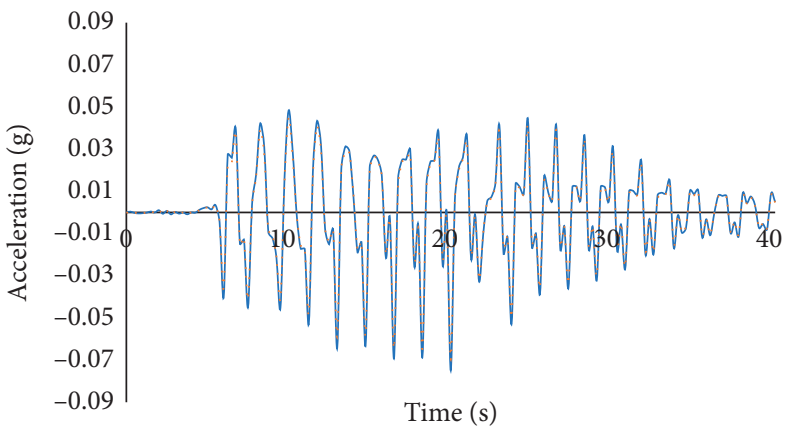

_ First method of calibration Third method of calibration

(b)

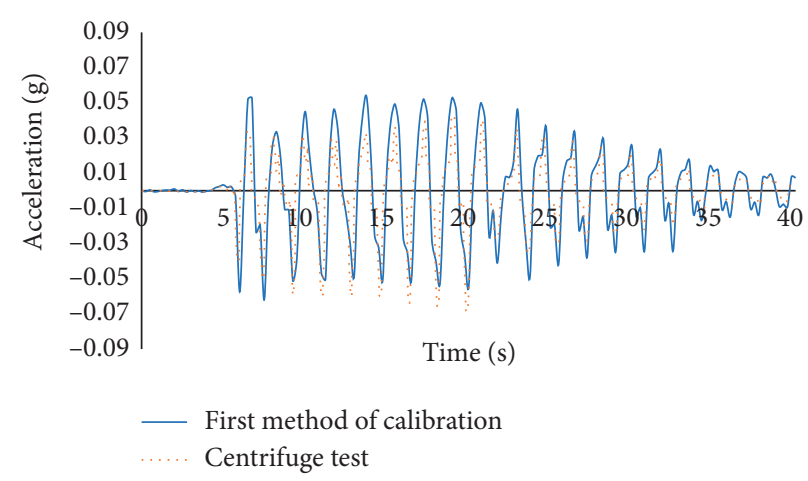

(d)

Figure 9: Continued. 


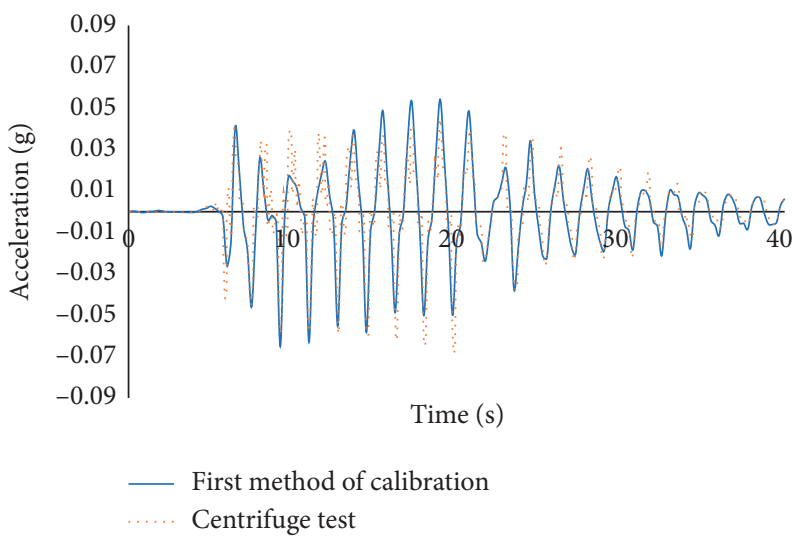

(e)

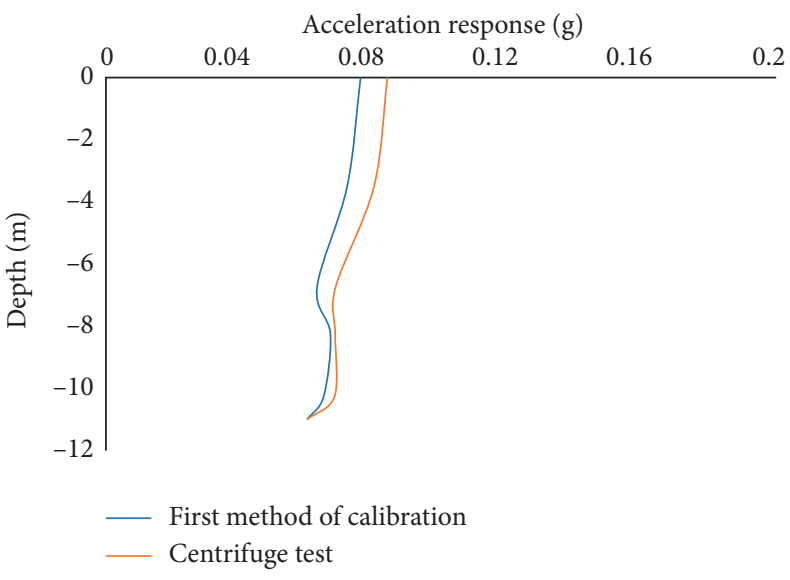

(f)

Figure 9: Maximum acceleration response of input motion-1 along the clay: (a) soil surface (numerical results), (b) soil surface (numerical results), (c) soil surface (numerical and the centrifuge results), (d) depth $=6.75 \mathrm{~m}$, (e) depth $=10.25 \mathrm{~m}$, and (f) soil depth.

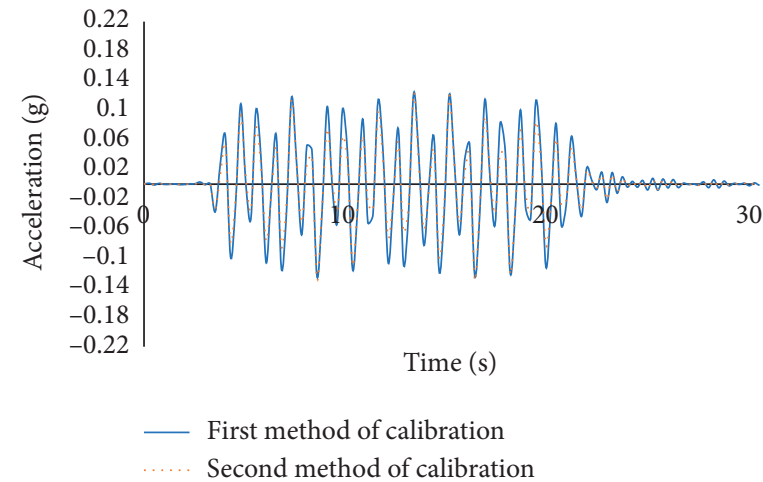

(a)

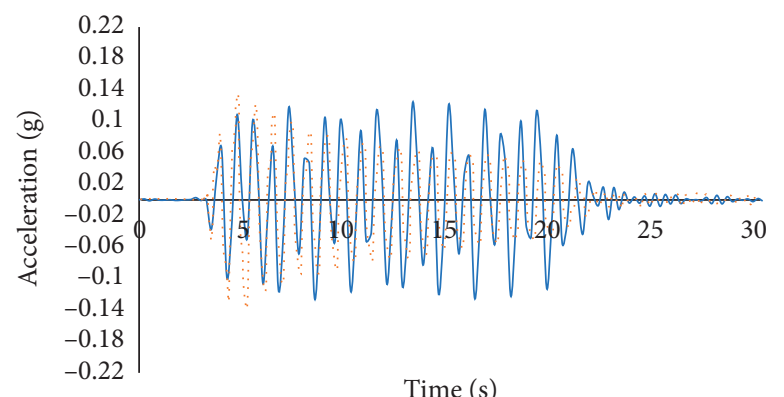

Time (s)

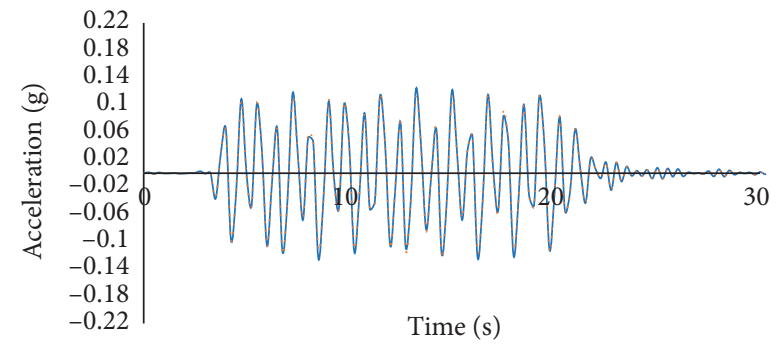

First method of calibration Third method of calibration

(b)

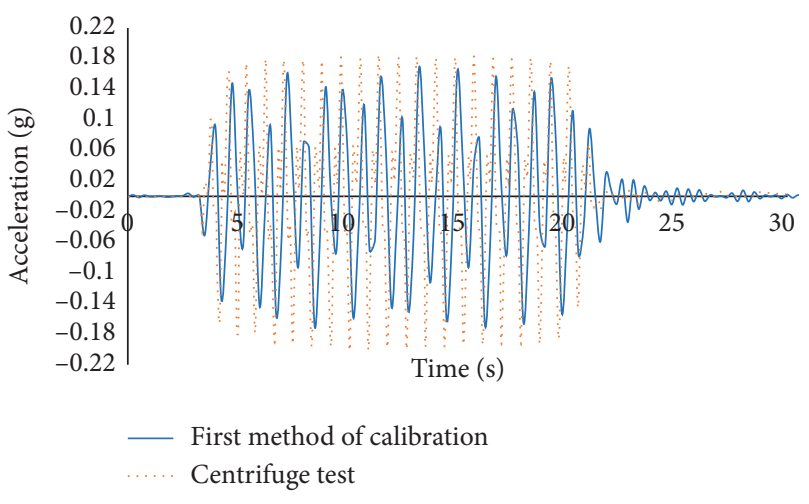

(c)

Figure 10: Continued. 


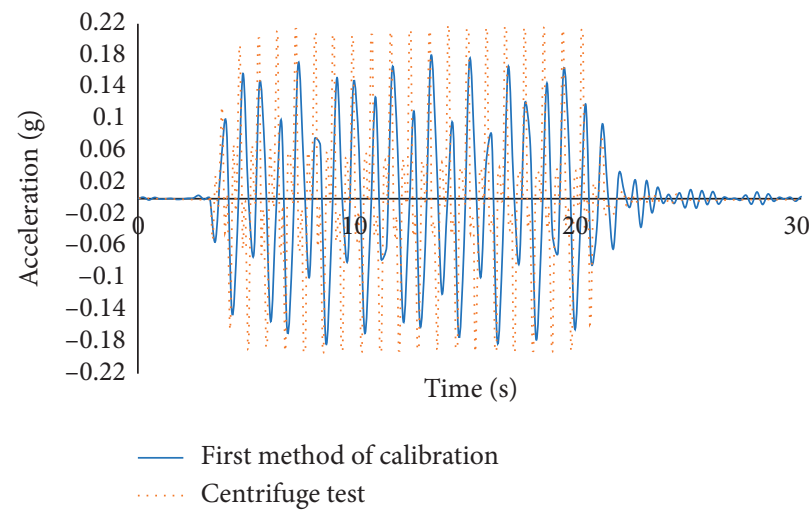

(e)

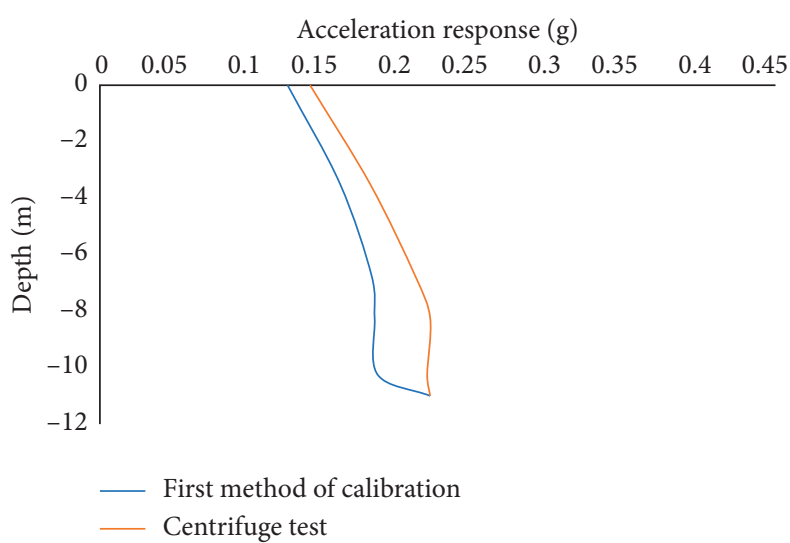

(f)

Figure 10: Maximum acceleration response of input motion-3 along the clay: (a) soil surface (numerical results), (b) soil surface (numerical results), (c) soil surface (numerical and centrifuge results), (d) depth $=6.75 \mathrm{~m}$, (e) depth $=10.25 \mathrm{~m}$, and (f) soil depth.

the natural frequency of models is close to the frequency of input motion-1. The maximum acceleration response on the soil surface of the centrifuge model is $0.084 \mathrm{~g}$, and in the numerical models based on the parameters obtained from the first, second, and third methods they are $0.076 \mathrm{~g}, 0.078 \mathrm{~g}$, and $0.07 \mathrm{~g}$, respectively.

The results of the pile-soil model analysis under medium frequency earthquake load $(0.8 \mathrm{~Hz})$ were also similar to those of the previous case. In the following, in order to investigate the effect of earthquake intensity (the results of the centrifuge model in both the lowest and highest earthquake frequencies are presented in the reference article [15]), the results of the pile-soil model under the earthquake load with the highest frequency $(1.2 \mathrm{~Hz})$ are presented in Figure 10. In this figure, the results of the numerical models (all three methods mentioned in Section 2.2.) and the centrifuge model are compared. As can be seen from the results, the acceleration response increases with increasing depth; this means that clay under high-frequency earthquake reduces the earthquake acceleration because the natural frequency of the model is not close to the frequency of input motion-3 so that the lowest amount of the maximum acceleration response is on the soil surface. This value was $0.14 \mathrm{~g}$ in the centrifuge model and $0.125 \mathrm{~g}, 0.13 \mathrm{~g}$, and $0.12 \mathrm{~g}$ in the first, second, and third methods of the numerical models, respectively. Thus, clay under the higher intensity earthquake decreases the acceleration response at the soil surface.

Figure 11 shows the acceleration response of the single pile, pile group-1, and pile group- 2 under the low-intensity earthquake (input motion-1). In all graphs, the acceleration response in the piles was increased relative to the input earthquake acceleration. In this figure, the results of the numerical model using the first calibration method were used to compare with the centrifuge model results. As Figure 11 shows, the acceleration response of the piles was modified compared to the input motion and, in all structures including single pile, pile group-1, and pile group-2, was more intensified than the acceleration response at the soil surface (see Figure 9). The interaction between soil and structure increases by decreasing piles spacing in a pile group, which will usually lead to an increase in acceleration response in model. It is worth noting that the reduction or increase of the acceleration response, in addition to the distance between piles in a pile group, depends on other parameters such as soil characteristics, earthquake intensity, etc. According to all figures shown above, there are little differences between the results of the numerical method and the centrifugal model. Therefore, the nonlinear kinematic hardening constitutive model is a suitable model for studying the seismic behavior of soil.

The bending moment created in the piles during earthquake loading is affected by various factors. Figure 12 shows the variation of bending moment over the duration of the earthquake for different depths of the single pile. As shown in the figure, the time of maximum bending moment creation (at the twentieth second) is the same at different depths of pile, due to the maximum amount of earthquake force applied to the model at this time, but their values are different for various reasons, such as varying earthquake acceleration at different depths, decreasing bed reaction coefficient, and deformation of the soil environment. The graphs in Figure 12 are the results of the analysis of the soilpile-raft model under the lowest intensity of the earthquake (input motion-1). In general, the bending moment of the pile decreases as the earthquake frequency increases, because by increasing the frequency the total displacement amplitude of the soil particles decreases, so fewer forces are imposed on the pile and the bending moments are also reduced. Also, as the natural frequency of soil-pile system approaches the earthquake frequency, the forces and bending moments created along the pile change. In this study, due to the characteristics of soil, piles, cap, and placing of structures in the soil, the value of bending moments is reduced by increasing the frequency of earthquake. Figure 13 shows the bending moment variations along the single pile under the three different earthquakes. These values decrease as the frequency of the earthquake increases 


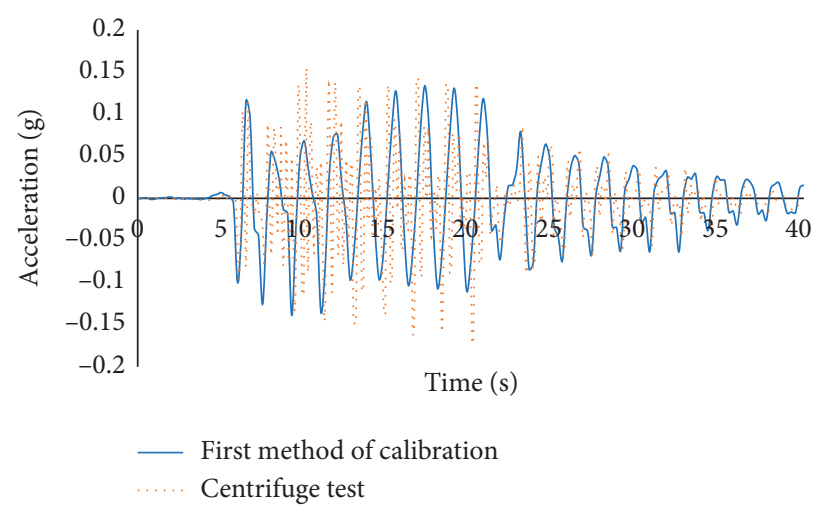

(a)

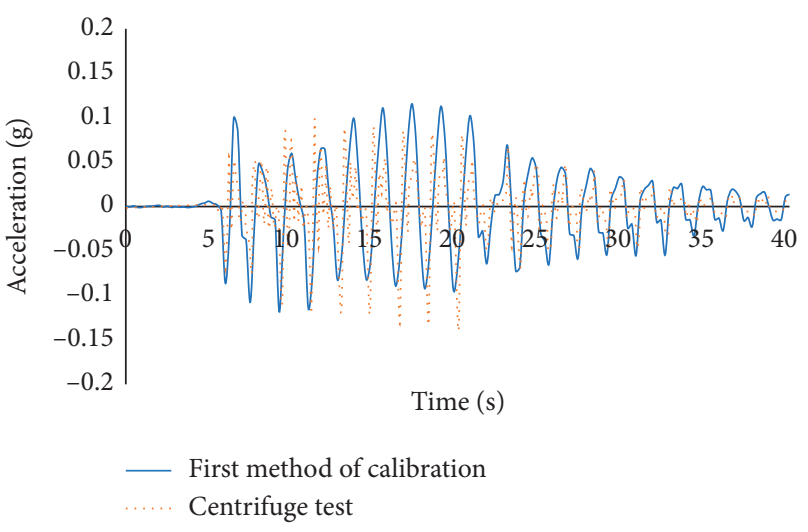

(b)

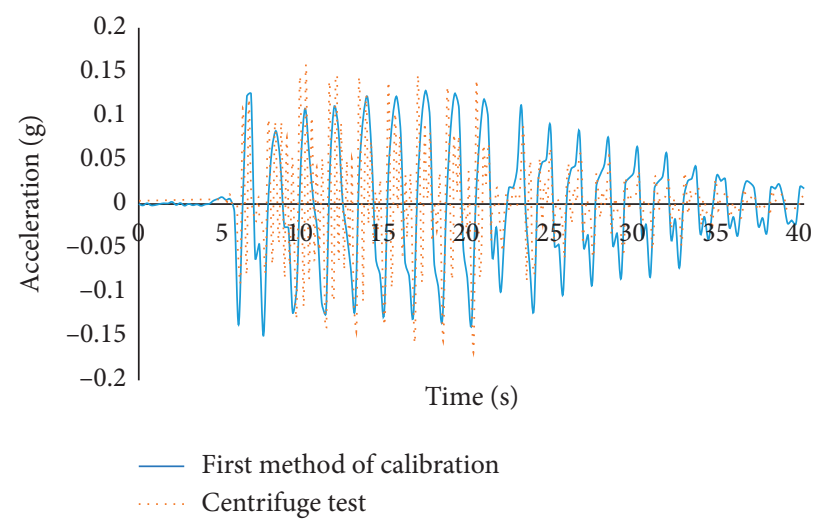

(c)

Figure 11: Maximum acceleration response of pile under input motion-1: (a) single pile, (b) pile group-1, and (c) pile group-2.

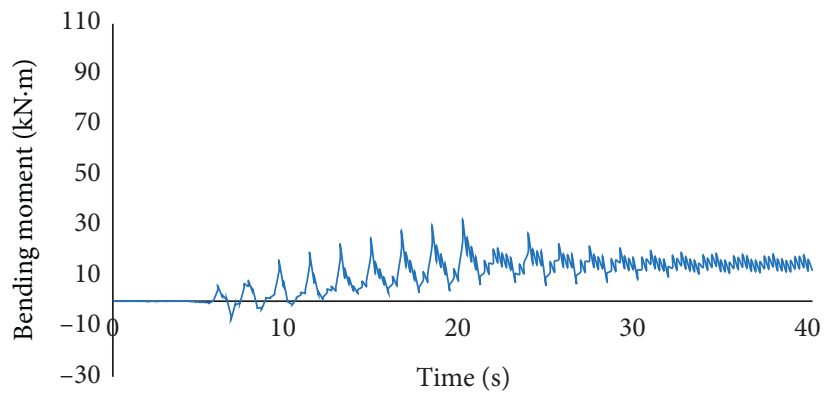

(a)

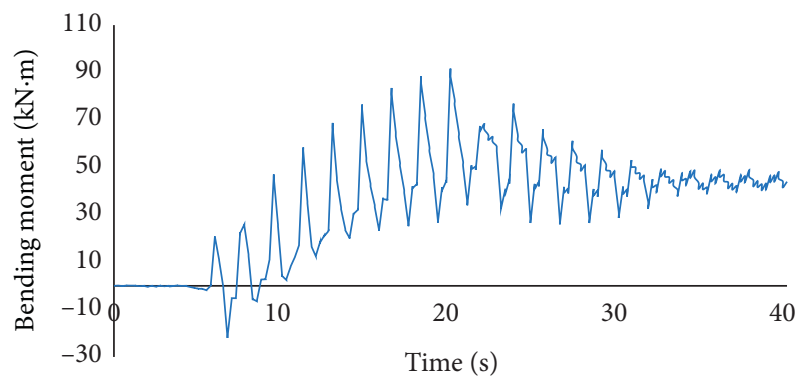

(b)

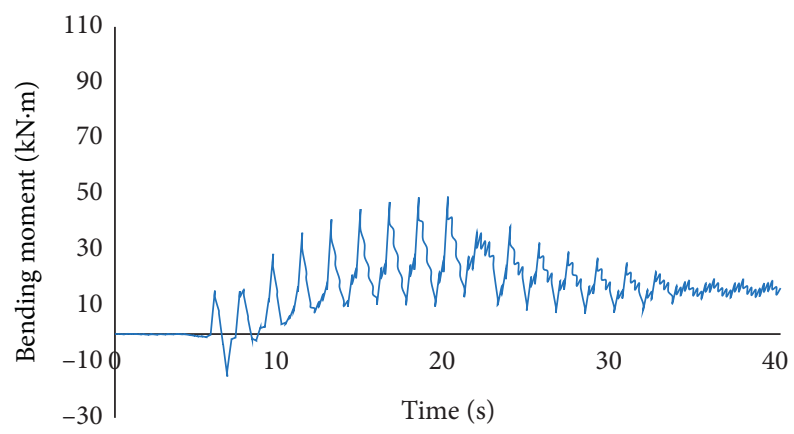

(c)

FIGURE 12: Bending moment of the single pile under the input motion-1 at different depths of pile: (a) $D_{1}=3 \mathrm{~m}$, (b) $D_{1}=6 \mathrm{~m}$, and (c) $D_{1}=9 \mathrm{~m}$. 


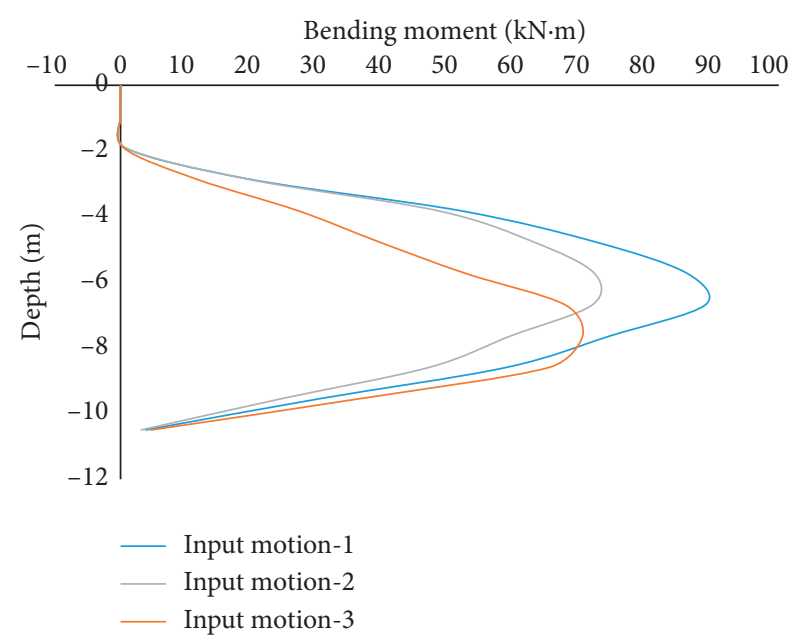

Figure 13: Bending moment variations along the single pile.

from $0.6 \mathrm{~Hz}$ to $1.2 \mathrm{~Hz}$. Figures 12 and 13 are the results of models where soil parameters have been calibrated by the first method.

\section{Conclusions}

In this paper, the seismic behavior of the pile-soil system under three different earthquakes was investigated; then, the results of the numerical analysis were compared with the results of the centrifuge model. In all analyses, the aluminum material considering the linear elastic model has been used for the piles, and an advanced nonlinear kinematic hardening model has been considered for soft kaolin clay. Three different calibration methods have been used to calibrate the constitutive model parameters where the second and third methods are the new methods presented in this study and the results of the numerical models were in good agreement with the results of the centrifuge model. All analyses were performed in $3 \mathrm{D}$ using Abaqus software. The results of this study are presented as follows:

(1) After comparing the results of the numerical analysis performed in this paper with the results of the centrifuge model, it can be concluded that the nonlinear kinematic hardening model is a suitable constitutive model for investigating the seismic behavior of the soil-pile-raft system.

(2) The nonlinear kinematic hardening model for clay soils that are under the cyclic loading and unloading can create hysteresis loops and is also able to show the increasing of damping caused by the soil plasticity, decreasing stiffness and soil strength by increasing the number of loading and unloading cycles.

(3) The most important part of using constitutive models in the analysis is the calibration of its parameters, so that the calibration method of constitutive model parameters has a great impact on the performance of the model and results of the numerical analysis. Here, three different methods were used to calibrate the parameters and the results were acceptable. But the results of the first and second methods were closer to the results of the centrifuge model.

(4) The decrease or amplification of earthquake acceleration in clay during the seismic loading mainly depends on the frequency and intensity of input earthquake acceleration and soil stiffness. According to the results of this research, the acceleration response is usually amplified in soft soil under the lowfrequency earthquake. So, the natural frequency of the system is close to the frequency of the low-frequency earthquake. Thus, the maximum acceleration on the soil surface is larger than the acceleration of the input earthquake.

(5) The distance between piles in a pile group has a great impact on the amount of interaction between them, acceleration response, forces, and bending moments. The interaction between soil and structure increases by decreasing piles spacing in a pile group, which will usually lead to an increase in acceleration response in model.

(6) Several factors affect the amount of bending moment created in the pile under the seismic loading. For example, it can be altered by changing the frequency and intensity of the earthquake, so that the bending moment created along the pile increases by decreasing the frequency. In this study, due to the characteristics of soil, piles, cap, and placing of structures in the soil, the value of bending moments is reduced by increasing the frequency of earthquake.

\section{Appendix}

\section{A. Effective Maximum Yield Stress for Clay Considering Pure Shear Test}

The second invariant of deviatoric stress tensor $\left(J_{2}\right)$ is expressed as follows:

$$
\begin{aligned}
& \frac{1}{6}\left[\left(\sigma_{11}-\sigma_{22}\right)^{2}+\left(\sigma_{22}-\sigma_{33}\right)^{2}+\left(\sigma_{33}-\sigma_{11}\right)^{2}\right] \\
& \quad+\sigma_{12}^{2}+\sigma_{23}^{2}+\sigma_{13}^{2}=k^{2} .
\end{aligned}
$$

By considering the main stresses,

$$
\frac{1}{6}\left[\left(\sigma_{1}-\sigma_{2}\right)^{2}+\left(\sigma_{2}-\sigma_{3}\right)^{2}+\left(\sigma_{3}-\sigma_{1}\right)^{2}\right]=k^{2} \text {. }
$$

Under the simple tension (or compression) conditions,

$$
\sigma_{2}=\sigma_{3}=0, \sigma_{1}=\sigma_{\mathrm{y}},
$$

where $\sigma_{\mathrm{y}}$ is the maximum yield stress in uniaxial tension or compression. By considering equations (A.2) and (A.3),

$$
\frac{1}{3} \sigma_{1}^{2}=k^{2}=\frac{1}{3} \sigma_{\mathrm{y}}^{2} \Rightarrow k=\frac{\sigma_{\mathrm{y}}}{\sqrt{3}} \text {. }
$$

According to equations (A.1) and (A.4), 


$$
\begin{aligned}
& \frac{1}{6}\left[\left(\sigma_{11}-\sigma_{22}\right)^{2}+\left(\sigma_{22}-\sigma_{33}\right)^{2}+\left(\sigma_{33}-\sigma_{11}\right)^{2}\right] \\
& +\sigma_{12}^{2}+\sigma_{23}^{2}+\sigma_{13}^{2}=\frac{\sigma_{\mathrm{y}}^{2}}{3}
\end{aligned}
$$

The pure shear condition is considered in order to evaluate the performance of the yield criterion, by replacing the stress tensor components in equation (A.5):

$$
\begin{aligned}
& \sigma_{11}=\sigma_{22}=\sigma_{33}=\sigma_{23}=\sigma_{13}=0, \\
& \sigma_{12}=S_{\mathrm{u}} \\
& S_{\mathrm{u}}=\frac{\sigma_{\mathrm{y}}}{\sqrt{3}} \Rightarrow \sigma_{\mathrm{y}}=\sqrt{3} S_{\mathrm{u}} .
\end{aligned}
$$

\section{Data Availability}

The data used to support the findings of this study are available from the corresponding author upon request.

\section{Conflicts of Interest}

The authors declare that there are no conflicts of interest regarding the publication of this paper.

\section{Acknowledgments}

The first author of this article is financially supported by Chinese Government Scholarship (CSC).

\section{References}

[1] J. Sun, S. Wang, X. Shi, F. Wu, and L. Zeng, "Study on the design method for the deformation state control of pile-anchor structures in deep foundation pits," Advances in Civil Engineering, vol. 2019, Article ID 9641674, 16 pages, 2019.

[2] L. Wang, Y. Zhou, W. Gong, J. Li, and A. Ishimwe, "Calculation method of seismic residual displacement of sheet pile quay walls," Advances in Civil Engineering, vol. 2019, Article ID 1251295, 15 pages, 2019.

[3] L. Zhang and H. Liu, "Seismic response of clay-pile-raft-superstructure systems subjected to far-field ground motions," Soil Dynamics and Earthquake Engineering, vol. 101, pp. 209224, 2017.

[4] G. Anoyatis, R. Di Laora, A. Mandolini, and G. Mylonakis, "Kinematic response of single piles for different boundary conditions: analytical solutions and normalization schemes," Soil Dynamics and Earthquake Engineering, vol. 44, pp. 183195, 2013.

[5] R. M. S. Maiorano, L. De Sanctis, S. Aversa, and A. Mandolini, "Kinematic response analysis of piled foundations under seismic excitation," Canadian Geotechnical Journal, vol. 46, no. 5, pp. 571-584, 2009.

[6] M. Saleh Asheghabadi and M. A. Rahgozar, "Finite element seismic analysis of soil-tunnel interactions in clay soils," Iranian Journal of Science and Technology, Transactions of Civil Engineering, vol. 43, no. 4, pp. 835-849, 2019.

[7] M. S. Asheghabadi and H. Matinmanesh, "Finite element seismic analysis of cylindrical tunnel in sandy soils with consideration of soil-tunnel interaction," Procedia Engineering, vol. 14, pp. 3162-3169, 2011.

[8] M. Patil, D. Choudhury, P. G. Ranjith, and J. Zhao, "A numerical study on effects of dynamic input motion on response of tunnel-soil system," in Proceedings of the 16th World Conference on Earthquake Engineering (16th WCEE 2017), Santiago, ChilePaper ID: 3313, Santiago, Chile, 2017.

[9] M. T. Rayhani and M. H. El Naggar, "Physical and numerical modeling of seismic soil-structure interaction in layered soils," Geotechnical and Geological Engineering, vol. 30, no. 2, pp. 331-342, 2012.

[10] C. c. Cremer, A. Pecker, and L. Davenne, "Cyclic macro-element for soil-structure interaction: material and geometrical non-linearities," International Journal for Numerical and Analytical Methods in Geomechanics, vol. 25, no. 13, pp. 1257-1284, 2001.

[11] T. Wichtmann, W. Fuentes, and T. Triantafyllidis, "Inspection of three sophisticated constitutive models based on monotonic and cyclic tests on fine sand: hypoplasticity vs. Sanisand vs. ISA," Soil Dynamics and Earthquake Engineering, vol. 124, pp. 172-183, 2019.

[12] P. J. Armstrong and C. O. Frederick, "A mathematical representation of the multi axial Bauschinger effect," vol. 24, no. 1, pp. 1-26, 2007, CEGB Report RD/B/N 731, Central Electricity Generating Board, Materials at High Temperatures.

[13] S. J. Zakavi, M. Zehsaz, and M. R. Eslami, "The ratchetting behavior of pressurized plain pipework subjected to cyclic bending moment with the combined hardening model," Nuclear Engineering and Design, vol. 240, no. 4, pp. 726-737, 2010.

[14] I. Ishibashi and X. Zhang, "Unified dynamic shear moduli and damping ratios of sand and clay," Soils and Foundations, vol. 33, no. 1, pp. 182-191, 1993.

[15] T. K. Garala, G. S. P. Madabhushi, and P. Madabhushi, "Seismic behaviour of soft clay and its influence on the response of friction pile foundations," Bulletin of Earthquake Engineering, vol. 17, no. 4, pp. 1919-1939, 2019.

[16] J. L. Chaboche, "A review of some plasticity and viscoplasticity constitutive theories," International Journal of Plasticity, vol. 24, no. 10, pp. 1642-1693, 2008.

[17] J. L. Chaboche and D. Nouailhas, "Constitutive modeling of ratcheting effects-part I: experimental facts and properties of the classical models," Journal of Engineering Material and Technology, vol. 111, p. 384, 1998.

[18] I. Anastasopoulos, F. Gelagoti, R. Kourkoulis, and G. Gazetas, "Simplified constitutive model for simulation of cyclic response of shallow foundations: validation against laboratory tests," Journal of Geotechnical and Geoenvironmental Engineering, vol. 137, no. 12, pp. 1154-1168, 2011.

[19] P. J. Armstrong and C. O. Frederick, "A mathematical representation of the multiaxial bauschinger effect," CEGB Rep. No. RD/B/N 731, 1966.

[20] N. Gerolymos and G. Gazetas, "Winkler model for lateral response of rigid caisson foundations in linear soil," Soil Dynamics and Earthquake Engineering, vol. 26, no. 5, pp. 347361, 2006.

[21] N. Gerolymos and G. Gazetas, "Development of Winkler model for static and dynamic response of caisson foundations with soil and interface nonlinearities," Soil Dynamics and Earthquake Engineering, vol. 26, no. 5, pp. 363-376, 2006.

[22] N. Gerolymos and G. Gazetas, "Static and dynamic response of massive caisson foundations with soil and interface nonlinearities-validation and results," Soil Dynamics and Earthquake Engineering, vol. 26, no. 5, pp. 377-394, 2006. 
[23] T. Wichtmann and T. Triantafyllidis, "Monotonic and cyclic tests on kaolin: a database for the development, calibration and verification of constitutive models for cohesive soils with focus to cyclic loading," Acta Geotechnica, vol. 13, no. 5, pp. 1103-1128, 2018.

[24] A. Zafeirakos and N. Gerolymos, "On the seismic response of under-designed caisson foundations," Bulletin of Earthquake Engineering, vol. 11, no. 5, pp. 1337-1372, 2013.

[25] G. Tsinidis, K. Pitilakis, and A. D. Trikalioti, "Numerical simulation of round robin numerical test on tunnels using a simplified kinematic hardening model," Acta Geotechnica, vol. 9, no. 4, pp. 641-659, 2014.

[26] K. H. Stokoe, M. B. Darendeli, R. D. Andrus, and L. T. Brown, "Dynamic soil properties: laboratory, field and correlation studies," in Proceedings of the 2nd International Conference on Earthquake Geotechnical Engineering, A.A. Balkema, Lisboa, Portugal, pp. 811-845, June 1999.

[27] M. Vucetic and R. Dobry, "Effect of soil plasticity on cyclic response," Journal of Geotechnical Engineering, vol. 117, no. 1, pp. $89-107,1991$.

[28] C. Smith, A. Kanvinde, and G. Deierlein, "Calibration of continuum cyclic constitutive models for structural steel using particle swarm optimization," Journal of Engineering $\mathrm{Me}$ chanics, vol. 143, no. 5, Article ID 04017012, 2017.

[29] M. Saleh Asheghabadi and X. Cheng, "Analysis of undrained seismic behavior of shallow tunnels in soft clay using Nonlinear kinematic hardening model," Applied Sciences, vol. 10, no. 8 , p. 2834,2020

[30] M. Saleh Asheghabadi and Z. Ali, "Infinite element boundary conditions for dynamic models under seismic loading," Indian Journal of Physics, vol. 94, pp. 907-917, 2019.

[31] H. Matinmanesh and M. Saleh Asheghabadi, "Seismic analysis on soil-structure interaction of buildings over sandy soil," in Proceedings of the Twelfth East Asia-Pacific Conference on Structural Engineering and Construction (EASEC-12), Hong Kong Special Administrative Region, Hong Kong, China, pp. 24-26, January 2011.

[32] M. Saleh Asheghabadi, M. Sahafnia, A. Bahadori, and N. Bakhshayeshi, "Seismic behavior of suction caisson for offshore wind turbine to generate more renewable energy," International Journal of Environmental Science and Technology, vol. 16, no. 7, pp. 2961-2972, 2018.

[33] Y. Li, M. Zhao, C.-s. Xu, X.-l. Du, and Z. Li, "Earthquake input for finite element analysis of soil-structure interaction on rigid bedrock," Tunnelling and Underground Space Technology, vol. 79, pp. 250-262, 2018.

[34] X. L. Du, Theories and Methods of Wave Motion for Engineering, Science Press, Beijing, China, 2009.

[35] L. Zhang, S. H. Goh, and H. Liu, "Seismic response of pileraft-clay system subjected to a long-duration earthquake: centrifuge test and finite element analysis," Soil Dynamics and Earthquake Engineering, vol. 92, pp. 488-502, 2017. 